\title{
Chitosan Applications on Pharmaceutical Sciences: A Review
}

\author{
Cintia Alejandra Briones Nieva, Mercedes Villegas, Alicia Graciela Cid, Analía Irma Romero and José María \\ Bermúdez
}

\begin{abstract}
Instituto de Investigaciones para la Industria Química, Universidad Nacional de Salta - Consejo Nacional de Investigaciones Cientificas y Técnicas, Av. Bolivia 5150, Salta Capital (4400), Argentina; Facultad de Ingeniería, Universidad Nacional de Salta, Av. Bolivia 5150, Salta Capital (4400), Argentina
\end{abstract}

\begin{abstract}
Background: Chitosan (CS) is a biomaterial derived from chitin, known for its excellent biological properties. One of the most interesting features of CS is its potential for chemical derivatization, which makes it a versatile material and allows to expand its applications. In the last years, the interest on this polymer and its pharmaceutical applications has notably increased. This biopolymer is being widely studied for its interesting properties, such as bioadhesion, antimicrobial activity, biocompatibility, and biodegradability. Other promising properties of CS include its modulation of immunological response, hemostasis, and wound and bone healing activity.

Objective: In this work, a critical review is performed covering its conventional and novel applications, specially focused on pharmaceutical area, providing a clear picture of the current state of art to serve as a basis to direct future research in this field.

Conclusion: Despite all the qualities of this polymer, there are only few CS-based products in the market, so it is a priority to enhance the research to develop new technologies and CS-based systems to enforce this biopolymer in the industry.
\end{abstract}

Received: December 21, 2018

Revised: March 01, 2019

Accepted: March 15, 2019 DOI: $10.2174 / 2210303109666190404143906$

Keywords: Biomaterials, pharmaceutical applications, biodegradable, biocompatible, drug delivery/release, gene therapy.

\section{INTRODUCTION}

A biomaterial is defined as any material or combination of materials, of natural or synthetic origin, designed to interact with the biological systems in order to evaluate, treat or replace some tissue or organ of living beings [1]. Biomaterials can be classified as natural or synthetic. The former, such as purified collagen and protein fibers, are complex, heterogeneous materials, and, in some cases, more difficult to characterize and process. Synthetics can be metals, ceramics or polymers, and are commonly known as biomedical materials, to differentiate them from those of natural origin [2].

In recent years, several biomaterials have been studied not only for medical and pharmaceutical uses, but also for agricultural and biotechnological purposes since they do not have toxic effects on the environment [3]. Within biomedical materials, biopolymers have particularly found several applications in the biomedical field. For example, alginate, chitosan (CS) and gelatin are being studied in regenerative medicine. Among the biopolymers, carbohydrate polymers have had a great impact in therapeutic, pharmaceutical and biomedical applications. Within this category, polysaccharides

*Address correspondence to this author at the Av. Bolivia 5150. Salta Capital (4400), Argentina; Tel: +54-387-4255410; Fax: +54-387-4251006; E-mail: josemariabermudez@gmail.com; jbermudez@unsa.edu.ar are one of the most abundant industrial raw materials, which have been the subject of intense research due to their biodegradability, biocompatibility, sustainability and biosafety. The second most abundant polysaccharide in nature after cellulose is chitin, from which CS is obtained (Fig. 1). Chitin is a white, hard, inelastic linear polymeric carbohydrate, composed of repeating units of N-acetyl-D-glucosamine and it is found in the exoskeleton of crustaceans, in the internal structure of invertebrates and in fungal cell walls.

Despite CS favorable biological and structural properties, its extensive use in biomedical applications is limited by its insolubility in water and in common organic solvents [4]. Nevertheless, in the last decade, remarkable advances have been made in CS and its derivatives, demonstrating their wide applications in the biomedical field. They have been exploited in the manufacture of immediate-release tablets, where they can act as a disintegrant [5] or as a binder [6], depending on the degree of deacetylation (DD) and the concentration employed in the formulation.

CS is also promising due to the presence of a large number of hydroxyl and amino groups, through which, it can be modified by various chemical reactions to prepare a series of CS derivatives as non-toxic biocompatible biomaterials [7]. An intensive research has been carried out to demonstrate the safety of the polymer and its ability to activate wound 

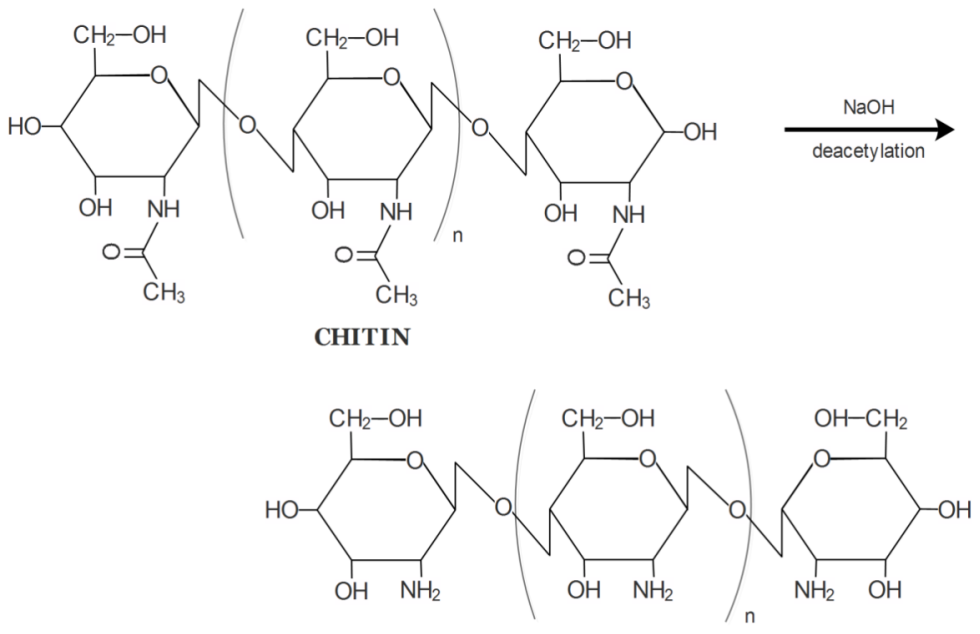

CHITOSAN

Fig. (1). Preparation of chitosan by the deacetylation of chitin.

Table 1. Some commercially available wound CS-based products.

\begin{tabular}{|c|c|c|}
\hline Commercial Name & Active Components & Presentations $^{\text {Patch, bandage, gauze }}$ \\
\hline \hline HemCon $^{\circledR}$ & Chitosan acetate & Roll, strip \\
\hline ChitoFlex $^{\circledR}$ & Chitosan acetate & Bandage \\
\hline TraumaStat $^{\circledR}$ & Chitosan & Nonwoven dressing \\
\hline Chitopack C $^{\circledR}$ & Chitosan acetate $^{\text {Films }}$ \\
\hline Tegasorb $^{\mathrm{TM}}$ & Chitosan & \\
\hline
\end{tabular}

healing and tissue repair. Furthermore, CS is generally recognized as safe [8] by the FDA for wound applications [9] and it has a well-characterized biocompatibility profile, which makes it a promising biopolymer for use in implanted devices and for drug delivery $[10,11]$. In fact, the development of commercial products in the form of wound dressings constitutes one of the most consolidated applications of this polymer in clinical practice (Table 1) [12-14].

Drugs such as antibiotics or antimicrobials may be mixed directly into viscous CS solutions which can then be used to develop films, beads, gels or other drug delivery devices [15]. Due to its positive charge, this polymer can interact with excipients and/or drugs with negative charge. This interaction has been intensively explored to modulate the release rate of low and high molecular weight (MW) drugs [16]. In the latter case, CS has been a powerful tool, allowing the administration of therapeutic proteins [17], vaccines [18], and DNA [19]. However, post-processing steps, that usually involve neutralization, cross-linking, rinsing, etc., can lead to the loss of the incorporated drug or drug activity.

Due to its excellent and unique biological properties (Fig. 2), CS is generally used as an antibacterial, antifungal and adhesive agent, either alone or blended with other polymers [4]. In fact, CS commercial applications are based on its antimicrobial and bio-stimulating properties. CS antimicrobial activity has been recognized for more than 30 years, although its precise mechanism of action remains unknown [20]. Probably, it is related to CS polycationic structure, since when the $\mathrm{pH}$ of the environment is below its $\mathrm{pKa}$, electrostatic interactions can occur between its polycationic structure and lipopolysaccharides and/or proteins in the surface of microorganisms, leading to the leakage of intracellu-

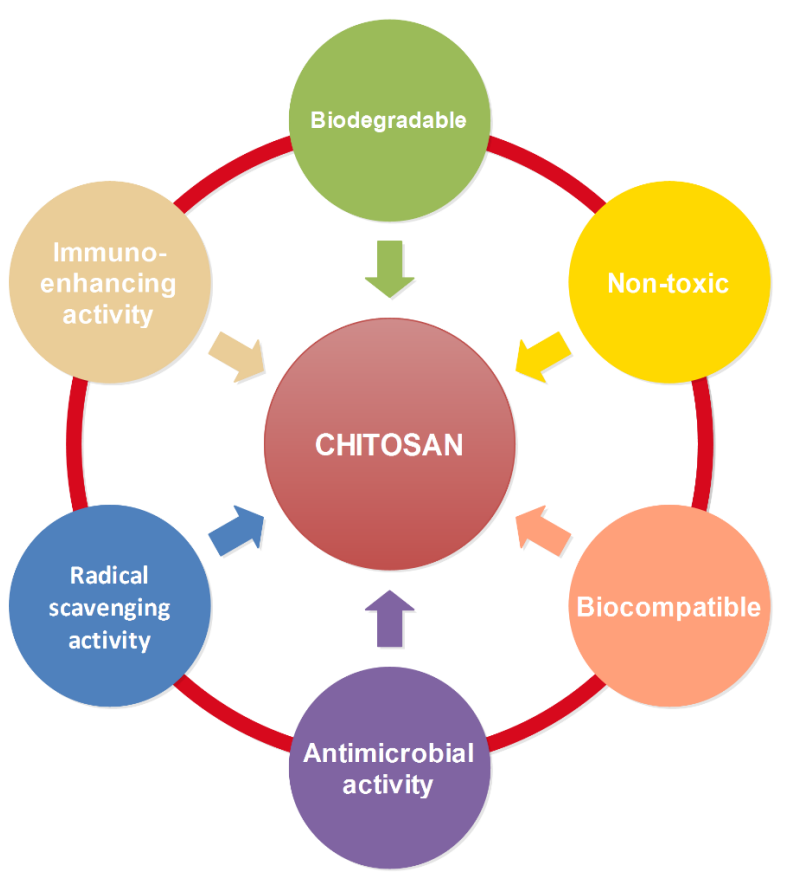

Fig. (2). Properties of chitosan. 
lar constituents [21]. Then, the antimicrobial activity is strongly influenced by the $\mathrm{pH}$ of the medium, but it also depends on CS MW and DD, and on the nature of the organism. In general, molds and yeasts are the most sensitive, followed by Gram-positive and Gram-negative bacteria [22]. As can be seen, CS has functional groups sensitive to the conditions of the surrounding environment, such as temperature, $\mathrm{pH}$, ionic strength, magnetic field, and ultraviolet light. These materials, commonly referred to as "smart materials" or "stimuli response materials", have promising applications in the area of drug delivery and wound healing.

Figs. (3 and 4) show the growing interest in CS represented by the number of scientific articles on "chitosan $\&$ biomedical applications" and "chitosan \& drug delivery", respectively, in the most recognized scientific publications databases available on the web, such as Science Direct, Wiley, Pub Med and ACS Publications.

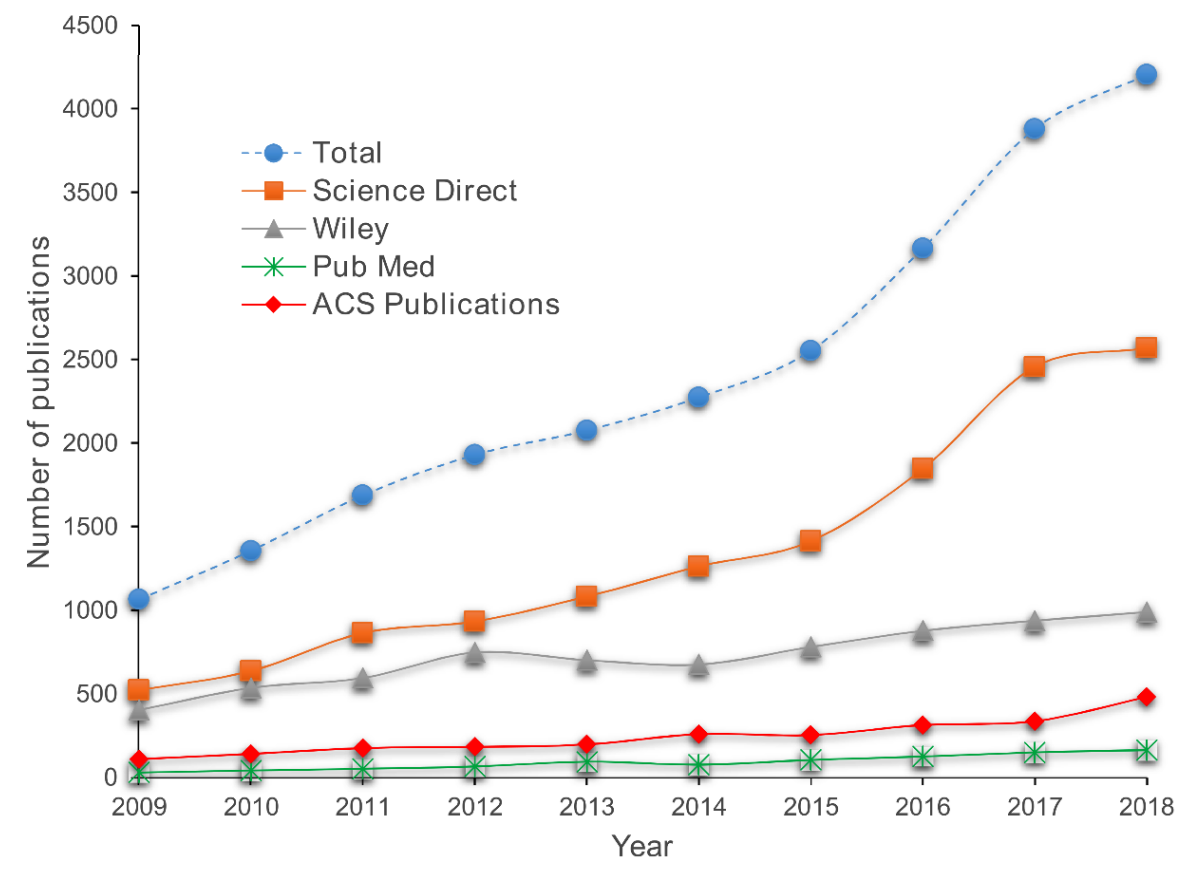

Fig. (3). Number of publications per year found with the keywords "chitosan \& biomedical applications" in scientific publication databases.

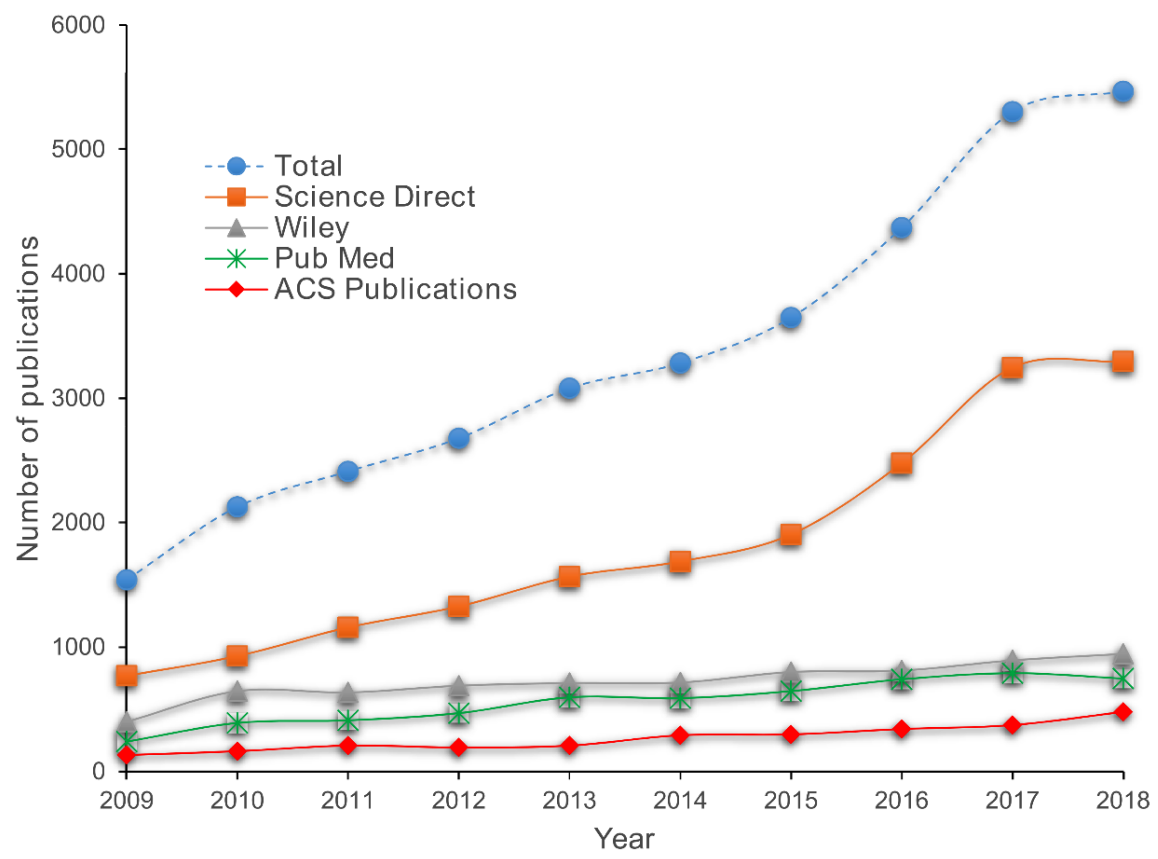

Fig. (4). Number of publications per year found with the keywords "chitosan \& drug delivery" in scientific publications databases. 

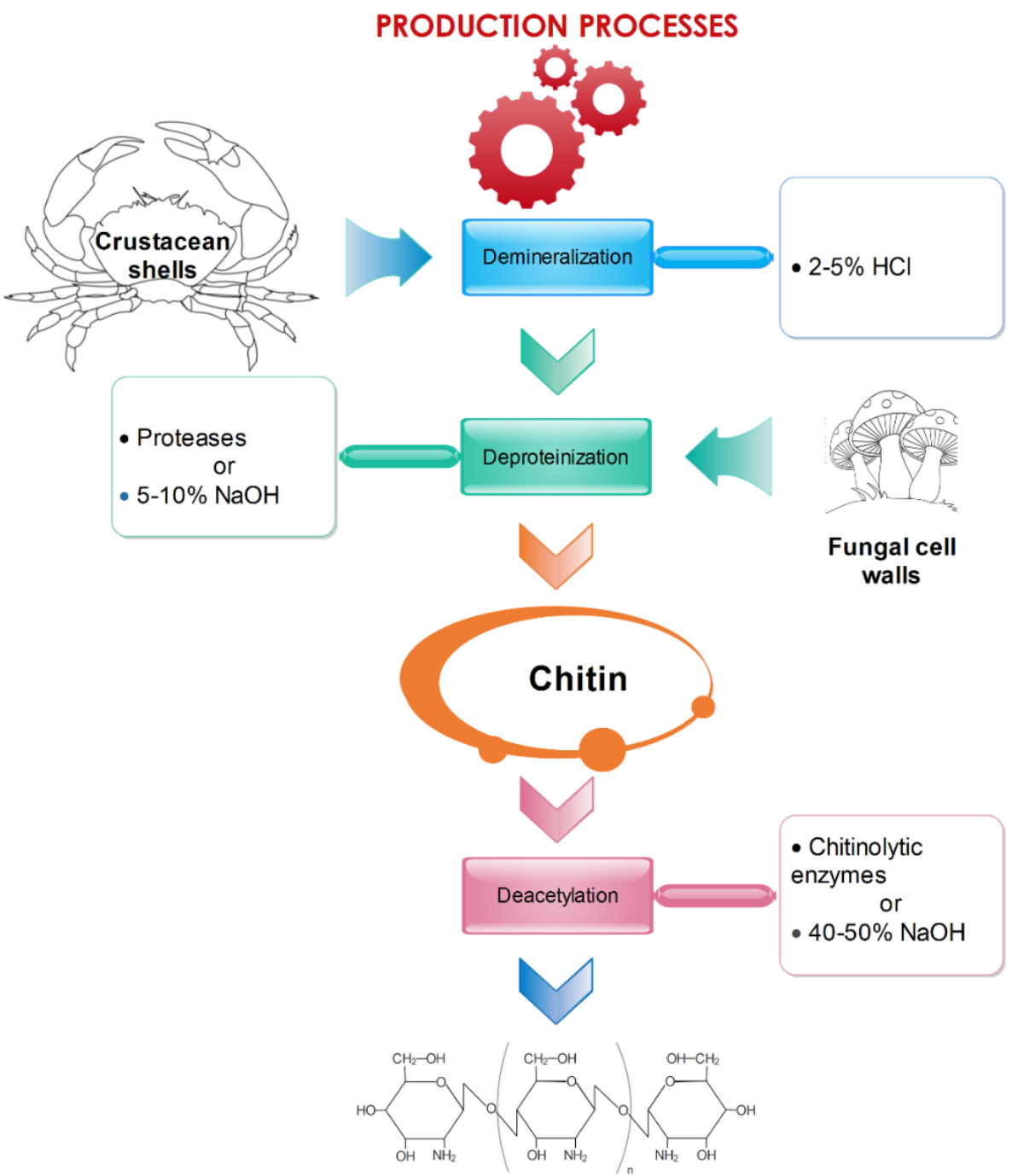

\section{CHITOSAN}

Fig. (5). Chitin and CS production by chemical and biotechnological treatments.

In this work, a critical review is performed covering CS conventional and novel applications, specially focused on the pharmaceutical area, providing a clear picture of the current state of the art to serve as a basis to direct future research in this field.

\section{CS PRODUCTION AND MODIFICATIONS}

CS is commercially obtained by deacetylating chitin, which is extracted either from crustacean shells via chemical and/or enzymatic procedures, or from mycelia obtained from fungal. Crab, shrimp, and prawn shells are the main raw material for chitin extraction, after which demineralization and deproteinization steps are required, followed by a deacetylation stage to transform the chitin into CS (Fig. 5). There are different alternatives to carry out these steps, including chemical and enzymatic procedures. Since the former involves the use of strong alkali and acids, they present some disadvantages, such as the polymer hydrolysis, the lack of reproducibility of the final physiological properties, and environmental pollution, making it necessary to further neutralize and detoxify the wastewater [23]. The replacement of one or several stages by biotechnological methods, including the use of enzymes, avoids some of these drawbacks, however, there are also some disadvantages associated with this technology, such as high residual protein, longer reaction times and high costs of enzymes, limiting the enzymatic methods for industrial applications [24]. One of the most developed alternatives in this sense is the raw material digestion with proteolytic enzymes such as papain, pepsin, trypsin, and pronase, during the deproteinization stage.

Once the chitosan is obtained, it can be further processed in order to grant certain characteristics. Fig. (6) summarizes some techniques used for the modification of CS, which include physical, chemical and electro-physical methods.

Among the possible physical modifications, cryo-milling is an effective method to produce micro-sized or even nanosized particles increasing their surface volume ratio [25]. However, one of the most interesting features of CS is its potential for chemical derivatization, which makes it a versatile material and allows to improve its solubility and to expand its applications. The hydroxyl groups at $C(6)$ and $C(3)$ positions and the amino or amido groups at $\mathrm{C}(2)$ positions 

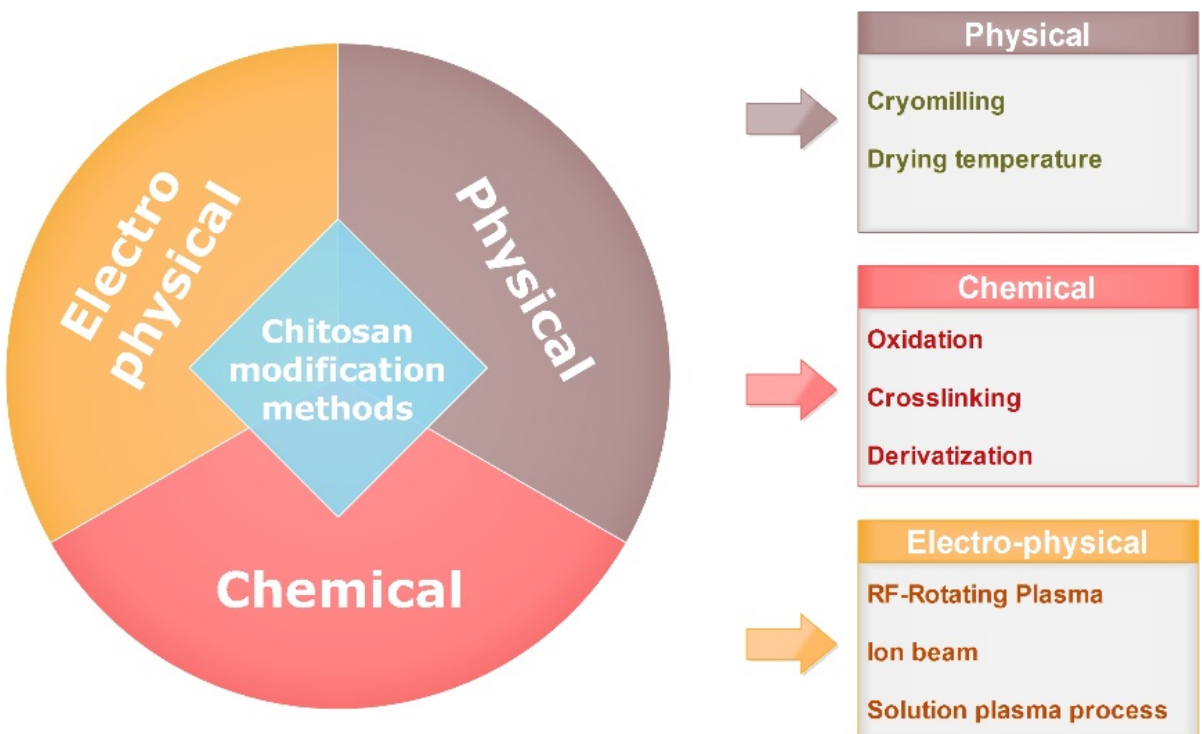

Fig. (6). CS modification techniques.

provide functional sites for specific modifications. Different chemical modifications were explored to prepare CS derivatives for biomedical applications, including deacetylation, Nacetylation and other acylation reactions, Schiff's base formation, N-phthaloylation, N-carboxyalkylation, N,Ocarboxymethylation, and graft copolymerization. Although CS chemical modification alters its properties, it is possible to preserve some important characteristics such as its biocompatibility, mucoadhesivity and biodegradability [26]. Chemical modification is a powerful tool to enhance drug load capability, to control polymer-drug interaction, and to improve CS bulk properties to prepare sustained drug release systems. The hydroxyl and amine groups of CS allow to attach CS coatings to different materials as well as to form antimicrobial derivatives [27]. Moreover, the antioxidant activity of CS and its derivatives is attributed to its ability to easily abstract hydrogen atoms from free radicals. The chemical modification of CS to produce new bio-functional materials has been successfully achieved [28]. Crosslinking is another method that can be employed to modify CS to change its hydrophilicity [29] or to increase its mechanical strength [30].

Finally, an interesting alternative to modify CS involves the use of electro-physical procedures. In this regard, Radio Frequency $(\mathrm{RF})$ rotating plasma, ion beam, solution plasma process, among others, offer some advantages over chemical methods such as oxidation, derivatization and crosslinking, since they are safe and clean processes [31], and avoid risks associated with chemical wastes.

\section{USE OF CS AND DERIVATIVES IN CONVEN- TIONAL PHARMACEUTICAL FORMULATIONS FOR BIOMEDICAL APPLICATIONS}

The existence of amino groups in the deacetylated CS provides functional binding sites to react with drugs, modulating the release profile of the active agent due to physical interactions [32-34]. Moreover, the biodegradability of CS is a very valuable property for designing drug delivery systems since surgical removal is not necessary after the therapeutic action has been completed [35]. On the other hand, the rheological properties of CS polymers also allow their use as an excipient, either as a binder or as a coating material in tablets [16]. In addition, the cationic charge of CS and the hydrogen bonding interactions give it mucoadhesive properties, which allow its adhesion to the mucin molecules that are found in the mucosal layer covering the epithelial tissues [36]. All these CS properties explain its wide range of applications.

\subsection{Oral Administration}

The oral route is the most frequently used in drug delivery for both conventional and novel drugs, due to the ease of administration and general acceptance by patients. However, the bioavailability of drugs after oral administration is very low, mainly because of their instability in the gastrointestinal (GI) tract, caused by the presence of peptidases and the strong acid $\mathrm{pH}$ in the stomach. In addition, a low permeability through the intestinal mucosa, in the case of drugs with high MW and/or hydrophilicity, will also contribute to low bioavailability. Controlled drug release systems for oral administration are generally based on polymers, and release mechanisms usually depend on the phenomena of diffusion, bioerosion or degradation, and swelling or generation of osmotic pressure. Diffusion process occurs when the drug-polymer system is exposed to the GI fluid, which causes the release of the drug from the polymeric carrier system to the surrounding medium. Depending on the composition of the drug-polymer complex, the phenomenon of bioerosion or degradation can occur in certain sections of the GI tract. The swelling phenomenon occurs mainly with polymers called polyelectrolytes when they are exposed to the GI fluid, which produces a modulated drug release, controlled by the material swelling rate.

The solubility of CS in an acidic environment can be exploited to develop coatings for advanced systems that respond to stimuli or are targeted to specific tissues. For example, a drug capsule can be coated with CS to prevent 
degradation at high $\mathrm{pH}$ until it reaches an acidic environment, such as in the stomach.

An interesting work was conducted by Gonçalves et al. [37], who used CS as a mucoadhesive coating on alginate beads loaded with amoxicillin. These beads exhibited excellent encapsulation efficiency, high drug loading capacity, and a strong in vitro mucoadhesion to the gastric mucosal layer. In the same regard, Wang et al. [38] formulated free liposomes of CS-coated cholesterol to encapsulate progesterone, based on the structurally similar properties of progesterone and cholesterol. The results obtained allowed them to conclude that CS-coated liposomes are a promising alternative to improve the oral bioavailability of progesterone.

In another interesting approach, Ubaid et al. [39] prepared an optimized CS/gelatin $\mathrm{pH}$-sensitive hydrogel using different concentrations of genipin as a crosslinking agent for the oral delivery of metformin. It was observed that the release rate profile could be modulated according to the concentration of the crosslinking agent.

\subsection{Buccal Mucosa Administration}

Drug administration through the buccal mucosa in the mouth provides unique advantages, such as avoiding the hepatic first-pass metabolism, and the acidity and proteolytic activity of the rest of the GI tract [40]. Buccal absorption of a non-ionized drug depends on its solubility in lipids, as well as on the $\mathrm{pH}$ of the saliva and the partition coefficient of the drug, which indicates its relative affinity for the vehicle and for the epithelial membrane. An ideal system for the buccal administration of drugs should comply with certain parameters, such as the ability to remain in the oral cavity for a few hours while releasing the drug unidirectionally towards the mucosa at a controlled or sustained rate. The drug delivery systems designed for buccal administration can be applied in the treatment of various diseases, like periodontitis, stomatitis, fungal and viral infections, and cancers of the oral cavity. Due to its muco/bioadhesive qualities, CS is an excellent candidate to transport buccal drugs, acting also as an absorption enhancer. In this regard, Freag et al. [41] evaluated the mucoadhesive properties of CS-based composite sponges with five different polymers: Carbopol $^{\circledR}$, sodium carboxymethylcellulose, hydroxypropylmethylcellulose, sodium alginate and hyaluronic acid. The formulations were prepared by adding each selected dissolved polymer in a CS hydrogel at different CS:polymer ratios, and then lyophilized. The authors concluded that all proposed composite sponges showed a percentage of moisture content and surface $\mathrm{pH}$ values within acceptable ranges. Moreover, among all the composite sponges, CS: hydroxypropylmethylcellulose (1:1) ones significantly enhanced the mucoadhesion time and showed the best physicochemical characteristics for buccal applications. Another study was conducted by Portero et al. [42], who developed a bilayered flexible device for the administration of insulin by casting/freeze-drying procedure, consisting in a mucoadhesive CS layer containing the peptide drug and an impermeable protective layer made of ethylcellulose. Three different salts of CS were compared: CS glutamate, CS tartrate and CS citrate. Authors found that the swelling properties of the sponges made from the different salts of CS were different and that the type of acid used to dissolve CS influenced insulin release from the sponges. They concluded that the affinity of chitosan sponges to mucin surfaces was related to the swelling and solubility properties of the different salts of chitosan.

Recently, Tejada et al. [43] described the formulation of miconazole nitrate buccal films and found that those formulated with CS-hydroxypropylmethylcellulose were the most appropriate due to their resistance to folding and their mechanical and mucoadhesive properties. In vitro antifungal activity assays showed a significant activity of the model drug used in the films.

\subsection{Gastro-retentive Delivery}

The gastro-retentive drug delivery systems are designed to increase the retention time in the stomach of a dosage form administered orally, representing an advantageous technological alternative for drugs that are absorbed in the GI tract, for drugs with limited solubility in the alkaline medium and also for drugs that are intended to produce a localized effect on the gastroduodenal wall [44]. They would be especially useful for drugs with limited solubility in the intestinal fluid and which are also highly soluble at acidic $\mathrm{pH}$. $\mathrm{CS}$ is a promising polymer for the development of gastroretentive drug delivery systems because it combines the properties of bioadhesion and flotation capacity [45]. CS could be ideal for the development of systems designed to release drugs at a slow rate in the stomach, due to the formation of a hydrogel, which will cause a delaying effect in the process of drug release.

In vitro and in vivo evaluation of gastro-retentive CS beads loaded with carvedilol were carried out by Praveen et al. [46]. The beads were found to have a plausibly and extended absorption due to the buoyant nature of the device in comparison with drug suspensions. On the other hand, in silico study indicated a maximum absorption from the duodenum (94.1\%) followed by jejunum. All these results allowed the authors to conclude that CS beads could be a potential drug carrier of carvedilol with improved bioavailability.

Kim et al. [47] designed bacteriophages belonging to the Myoviridae family encapsulated into CS-alginate microspheres to evaluate their acid stability, release property and antimicrobial efficacy against Escherichia coli under simulated GI conditions. They found that the bacteriophages were stable at $\mathrm{pH}$ values above 4 , but the CS-alginate microsphere exhibited a protective effect on the bacteriophages in the simulated gastric ( $\mathrm{pH} 2)$ conditions. Moreover, the encapsulated bacteriophages maintained the lytic efficacy against $E$. coli in the simulated intestinal conditions for $10 \mathrm{~h}$ of incubation, suggesting that these microspheres could be used as a reliable delivery system for bacteriophages.

\subsection{Colon-specific Drug Delivery}

Drug delivery systems directed to the colon have been widely studied in the last two decades. Although oral absorption occurs mainly in the small intestine, the residence time of the ingested materials in the colon is much higher. The peristaltic movements cause the food to pass through the small intestine in a few hours, but it can remain in the colon 
for up to 3 days. The colonic administration of drugs has several therapeutic advantages over the oral administration of drugs, wherein the drugs can be destroyed by stomach acid and/or metabolized by pancreatic enzymes. In this context, CS is widely used for the administration of colonspecific drugs, due to its high solubility in acidic stomach fluids, being degraded in the colon, like other polysaccharides. Although eventually, CS can become insoluble in an acidic environment by chemical crosslinking, what would be desired for a colon drug delivery system, this modified polymer is ineffective in preventing the release of the encapsulated drugs.

Drechsler et al. [33] developed film-coated tablets containing the model drug diclofenac-Na. The tablets consisted of a core of the drug and microcrystalline cellulose (MT), or Ludiflash $^{\circledR}$ (LT), and were coated with CS alone or different ratios of CS and Kollicoat (KCSS). CS/KCSS (25:75) coated LTs showed a pressure-controlled site-specific drug release in the large intestine while remaining intact in the upper GI tract. CS as well as CS/KCSS $(25: 75)$ applied onto MTs, remained stable during the entire simulated bio-relevant dissolution transit of the GI tract, but showed enzymatically controlled colon targeting in the colon microflora test. This result was confirmed for CS/KCSS (25:75) applied onto MTs with an additional hydroxypropylmethylcellulose layer and a Eudragit ${ }^{\circledR}$ layer to avoid the dissolution in the fasting stomach.

\subsection{Vaginal-specific Drug Delivery}

The current research on vaginal drug delivery is focused on the systemic delivery of drugs such as some hormones and prostaglandins. New technologies are evaluating the feasibility of providing therapeutic peptides and proteins through the vaginal route to achieve a systemic effect. The volume of fluid within the vaginal cavity is relatively small. For this reason, probably the limiting step in the process of systemic absorption of drugs transported in creams, inserts and intravaginal tablets is the rate of dissolution in the vaginal fluid. This is much more evident for drugs with limited solubility. In addition to the physicochemical factors associated with the drug, the stability of the drug in vaginal fluids is particularly important. Furthermore, labile drugs may undergo enzymatic deactivation before absorption.

Abruzzo et al. [48] prepared freeze-dried vaginal inserts of CS/alginate at different polycation/polyanion molar ratios and loaded with chlorhexidine digluconate for local delivery in genital infections. In vitro water-uptake, mucoadhesion and release tests showed that a suitable CS/alginate molar ratio and drug loading allowed to modulate the insert ability to hydrate, adhere to the mucosa, and release chlorhexidine digluconate. On the other hand, authors found that the insert containing an excess of alginate had the best performance and antimicrobial activity against the pathogens Candida albicans and E. coli. Other interesting research was conducted by Marciello et al. [49], who developed sponge-like systems consisting of CS nanoparticles (NPs) loaded with the model peptide insulin using mannitol, sucrose, gelatin B and polyethylene glycol (PEG) stearate as excipients. The objective of this study was to facilitate the in situ application and quickly dissolution in the vaginal environment, releasing the peptide-loaded NPs. In vitro and ex vivo experiments indicated that the designed systems were able to promote the peptide release and penetration into vaginal mucosa. On the other hand, the cylinders based on mannitol/sucrose and gelatin showed the best mechanical resistance properties for their application and storage.

\subsection{Nasal Administration}

Due to the high vascularization of the nasal mucosa and the possibility of avoiding the first hepatic passage, added to the ease of administration, the nasal route represents an interesting alternative for the recurrent administration of some drugs. The advance of biotechnology has increased the availability of proteins and peptides (for example, insulin, growth hormone, etc.), leading to growing research and development of innovative intranasal drug delivery systems [18]. Among the latest advances, mucosal vaccines for nasal administration stand out. CS has been used not only as an excipient in the formulation but also as a promoter of the absorption of therapeutic proteins administered nasally. It has been proved that the joint administration of CS and antigens in the nasal cavity produces an improvement of the systemic and also of the mucosal immune responses. Unlike oral administration, vaccines administered by the nasal route must be transported for short distances, in addition to not being exposed to low $\mathrm{pH}$ values and degrading enzymes. For this reason, it is not necessary to incorporate the vaccine into microparticles when administered nasally. Thus, CS particles, powders, and solutions are promising candidates for mucosal and nasal vaccine delivery. In this regard, Dabaghian et al. [50] evaluated a nasal vaccination with r4M2e.HSP70c influenza antigen encapsulated into $\mathrm{N}$ trimethyl CS (TMC) nanoparticulate systems. The results showed that intranasal immunization of mice with TMC NPs significantly increased the longevity and serum level of the total M2e-specific IgG antibody compared to the control groups.

When a rapid onset of action is required, the CS-based technology for nasal administration can provide a rapid maximum concentration, compared to oral or subcutaneous administration. The combination of bioadhesion and the opening of tight junctions between epithelial cells play a major role in CS-based mucosal delivery [51].

An interesting work was done by Luppi et al. [52], who developed a CS/pectin based nasal insert to improve the bioavailability of antipsychotic drugs. These polyelectrolyte complexes were prepared with different molar ratios of polycation/polyanion and lyophilized in small inserts in the presence of chlorpromazine hydrochloride. The results showed that a greater amount of pectin in the complexes produced a more evident porous structure of the nasal inserts, improving water uptake ability and mucoadhesion capacity, but the interaction between pectin and chlorpromazine-induced the formation of less hydratable inserts, limiting drug release and permeation.

\subsection{Pulmonary Delivery}

Since the mid-twentieth century, aerosols have been used in different ways for the treatment of respiratory disorders. However, interest in the pulmonary route for the systemic 
administration of drugs is relatively recent. The potential of this route for the administration of peptides and its viability for the treatment by gene therapy of cystic fibrosis have motivated researchers remarkably. The administration of drugs based on the inhalation is a practical and non-invasive way to administer therapeutic agents, including genes, to the lungs. However, a study found that CS microparticles used for inhalation induced dose-dependent proinflammatory effects in rat lungs [53]. In this sense, Zhang et al. [54] performed in vivo implantation experiments of theophylline/carboxymethyl $\mathrm{CS} / \beta$-cyclodextrin microspheres, founding that the inflammatory reaction gradually lessened and disappeared and had no significant difference from that of an operative suture. Manca et al. [55] developed new liposomes coated with CS-xanthan gum as potential carriers for pulmonary delivery of rifampicin. Results indicated that nebulization and rheological properties of coated liposomes were affected by the CS-xanthan gum weight ratio. Another interesting approach was conducted by Oyarzun-Ampuero et al. [56], who prepared mucoadhesive nanocarriers based on $\mathrm{CS}$ and hyaluronic acid, loaded with heparin. The nanocarriers presented a high drug loading efficiency (about $70 \%$ ) and high stability in phosphate-buffered saline. Moreover, ex vivo experiments indicated that these nanocarriers could be used for effective pulmonary delivery systems.

\subsection{Transdermal Administration}

Transdermal drug delivery systems have been widely used to release drugs through the skin and represent a valuable alternative to conventional routes of administration, such as oral, intravascular, subcutaneous and transmucosal. Many drugs have inherent side effects that cannot be avoided under any form of dosage. However, there are drugs that manifest undesirable effects that are specifically related to a particular route of administration. Different technological strategies are being used to avoid some of the problems related to traditional dosage forms, and one of the most promising is the development of transdermal drug delivery systems. In this regard, the development of a suitable formulation is essential to provide adequate and reproducible drug release rates. The components of the formulation will determine the rate of release of the drug in the skin and also in the substrate used for the adherence of the device to the skin, which will, therefore, influence the performance of the final product.

Anirudhan et al. [57] developed an efficient device based on CS and hyaluronic acid for the administration of transdermal lidocaine. The results indicated that this device had a good skin adhesion and did not irritate the skin even after 24 $\mathrm{h}$ of application. In addition, it was resistant to microbial activity and was suitable for long-term drug delivery.

Due to their unique properties, nanofibers have been studied in transdermal drug delivery systems, as they could provide constant blood levels over a longer period and avoid first pass metabolism. Cui et al. [58] evaluated electrospun fibers of cross-linked polyvinyl alcohol/CS (PVA/CS) loaded with ampicillin sodium for transdermal drug delivery. It was observed that the cross-linked PVA/CS composite nanofibers had lower drug release rate and a smaller amount of drug burst release than that of PVA/CS (without crosslinking).

\subsection{Ophthalmic Drug Delivery}

The ocular route for drug administration is used only for the local treatment of eye conditions and it is not viable to use it for the systemic administration of drugs. The efforts in the ocular administration of drugs have been oriented to improve the corneal penetration and the residence time of drugs in the eye, in order to increase the effectiveness of the treatments of different ocular diseases. The attempts include the use of colloidal drug delivery systems, such as liposomes, biodegradable NPs, and nanocapsules [59]. It should be noted that the short residence time of these colloidal formulations in the ocular mucosa is the main limitation in the treatment of extraocular diseases, such as keratoconjunctivitis sicca, or dry eye disease [59]. On the other hand, remarkable advances have been made in recent years to optimize the localized supply of drugs in the eye. In this way, the ocular route is now associated with the application of drug delivery technologies with a high level of sophistication. Some of these technologies are unique to the eye pathway and many of them are also used in other drug delivery routes. From the ocular point of view, the physicochemical properties of CS show a pronounced effect on drug stability within the ocular bioenvironmental conditions $[60,61]$. In addition, the positive charge and the bioadhesive nature of CS could allow increasing drug residence time in ocular tissues by ionic interaction with the negatively charged mucin residues located on the surface of the eye. CS-based carrier systems have been developed for the delivery of many ocular therapeutic molecules including antibiotics, anti-inflammatory, anticholinergics, analgesics, proteins and peptides, and genes, among others [62].

Natesan et al. [63] worked with CS NPs and PEGmodified CS NPs co-encapsulated with resveratrol (RES) and quercetin (QUR). They evaluated the synergic effect on reducing the intraocular pressure for glaucoma treatment in tests performed with normotensive rabbits. Authors reported a sustained and enhanced reduction of the intraocular pressure for the PEG-modified CS NPs loaded with RES and QUR. On the other hand, Rodriguez et al. [64] prepared dexamethasone loaded CS films, that were tested as a topical ocular delivery dosage form. The authors determined that the release time was longer than for conventional systems, making it an interesting option as an ocular delivery carrier.

\section{ADVANCED APPLICATION OF CS AND DERIVA- TIVES IN DRUG DELIVERY SYSTEMS}

$\mathrm{CS}$ is a promising biopolymer with a high potential for applications in the biomedical field due to its versatility and ability to form different configurations, including films, beads, sponges, and gels. In addition, CS can be chemically modified to functionalize it and improve its performance in the area of medicine, especially in relation to the drug delivery, for example, to design antibiotic release systems for local or systemic use $[65,66]$. CS can also bind to proteins, DNA, and RNA $[67,68]$, which may be useful in treating or preventing infections through vaccines or gene therapy [69]. Other advantages of CS include modulation of im- 
munological response, hemostasis, wound and bone healing activity, and inherent antimicrobial activity [10].

CS has been explored to form hydrogels for drug delivery because of its solubility in acidic solutions and its cationic charge in the protonated form [65]. Among the main advantages of CS hydrogels used for local drug delivery, injectability, rapid degradation, and elimination can be mentioned, as well as the possibility of achieving a controlled release of drugs. On the other hand, CS-based hydrogels have the potential to develop intelligent drug delivery systems, which respond to various stimuli, such as a change in $\mathrm{pH}$ or temperature, among others. The mechanism of drug release from hydrogels is generally governed by the diffusion step, but it can also be modified by modulating the swelling process that will lead to the erosion of the hydrogel.

\subsection{Stimuli-sensitive CS -based Systems}

In the biomedical area, special attention has been paid to the development of CS hydrogels sensitive to stimuli with biodegradability and biocompatibility qualities. An ideal drug delivery system that responds to different stimuli should be simple and inexpensive to manufacture, with the ability to deliver precise amounts of the drug in response to physiological or pathological stimuli. Polymeric hydrogels are defined as three-dimensional networks formed by trapping water molecules in their structure producing the swelling of the system. Due to their swelling properties, membranes based on these hydrogels are usually highly permeable to hydrophilic or high molecular weight agents. This property, added to the biocompatibility and biodegradability, has motivated the development of hydrogels that act as membranes in devices to control the rate of protein release, such as insulin, aprotinin, tumor antigen factor, and luteinizing hormone. According to the previously mentioned, the so-called "intelligent hydrogels" are of great interest because they can manifest a reversible discontinuous phase change in response to various external physicochemical factors. Among the most feasible stimuli to be used in in vivo applications, those that respond to changes in temperature and $\mathrm{pH}$ stand out.

\subsection{1. pH-sensitive CS-based Systems}

The chemical structure of CS and its derivatives shows that they contain hydroxyl and amino groups, and some of their derivatives can also have carboxyl or other functional groups. These ionizable groups are strongly influenced by the $\mathrm{pH}$ of the medium. For this reason, when an external $\mathrm{pH}$ change occurs, the dissociation equilibrium, the swelling characteristics and the interaction of CS and its derivatives with the solvents can be strongly modified [70]. Due to the unique characteristics of $\mathrm{pH}$ sensitivity, these systems have begun to find several applications in the biomedical and bioengineering fields, particularly as drug delivery carriers. Based on the CS own acidity, different CS-based systems have been prepared, including gels, interpenetrating polymer networks, NPs, and copolymers, among others, by adding different substances.

Du et al. [71] published a review article about CS-based $\mathrm{pH}$-responsive nanoformulations for GI delivery. The authors have summarized different architectures and physicochemical properties used in $\mathrm{pH}$-responsive systems for drug delivery in the GI tract.

Interestingly, Yilmaz et al. [72] have prepared CS-graft[poly(diethylamino)ethyl methacrylate] in three different physical forms, as linear free chains in solution, chemical gels crosslinked with glutaraldehyde, and poly(diethylamino)ethyl methacrylate grafted onto CS tripolyphosphate gel beads. They found that solubility of the products was controlled by the grafting yield. While $\mathrm{pH}-$ sensitive polymers, which collapse at a given $\mathrm{pH}$ value, were obtained at lower grafting yields, hydrogels formed at higher grafting yields with $\mathrm{pH}$-responsive swelling behavior. Glutaraldehyde crosslinked CS-graft-[poly(diethylamino)ethyl methacrylate] gels and CS tripolyphosphate gel beads grafted with poly[(diethylamino)ethyl methacrylate] exhibited $\mathrm{pH}-$ sensitive swelling with highest equilibrium swelling capacity at a $\mathrm{pH}$ value of 1.2.

\subsubsection{Thermo-sensitive CS-based Systems}

Thermo-sensitive polymers exhibit a temperaturedependent phase transition in solution across a critical temperature which is known as the critical solution temperature [70]. Copolymerization of CS or derivatives with some thermo-sensitive polymers, such as N-substituted polyacrylamides, methylcellulose, PVA, polyacrylic acid, polyvinylpyrrolidone, and polyethylene oxide, among others, can be used to produce new thermo-sensitive systems, which in turn have been used for obtaining copolymers, gels, beads, membranes, and NPs. Following this line, Li et al. [73] developed a thermo-sensitive injectable N-hexanoyl glycol CS hydrogel system for the percutaneous treatment of disc herniation to both restore the biomechanical function and reduce low back pain. The hydrogel showed a thermo-reversible solgel transition at low concentrations (3-7\%wt). In vitro and in vivo tests reported no cytotoxicity nor adverse effects in a rat model. The hydrogel filling of the defective IVD site in an $e x$ vivo porcine model maintained its stability for longer than 28 days, suggesting that this material can be an alternative for the treatment of disk herniation.

\subsubsection{Photo-sensitive CS-based Systems}

Photo-sensitive polymers undergo a macroscopic transition effect when submitted to irradiation. The introduction of photo-sensitizers in CS results in photo-sensitive polymers. In this regard, Jeong et al. [74] used water-soluble CS to fabricate chlorin e6 (Ce6)-incorporated nanophotosensitizers (ChitoCe6) via a self-assembling process. The photodynamic effect of ChitoCe6 was evaluated using GI cancer cells (SNU478 cholangiocarcinoma cells). The results showed that the particle accumulated effectively in the tumor tissue and inhibited tumor growth more than the treatment with Ce6 alone. Furthermore, the nanophotosensitizer was also efficiently absorbed through tissue layers in an ex vivo study using porcine bile duct explants.

\subsubsection{Electro-sensitive CS -based Systems}

Polymers sensitive to electric field undergo various changes in macroscopic behavior, such as those induced by electric current. Since CS is a polycationic polymer, an electrostatic interaction with polyacids or polyanions is possible. 
It is known that an ion can move in the opposite direction to the application of an electric field. To confirm it, Goycoolea and Milkova [75] adsorbed CS with different MW and DD onto lecithin stabilized nanoemulsion droplets. They investigated electrokinetic properties of the nanoemulsion by using dynamic light scattering, obtaining that the isoelectric point of the droplets in suspension in the presence of CS with opposite charge did not depend on the polyelectrolyte MW (when DD of CS was upper than $32 \%$ ). On the other hand, the stability behavior of the nanoemulsion in the presence of CS with lower charge density was different, and the isoelectric point and stabilization of the system were achieved at higher polymer concentration, agreeing with the notion that the electrostatic interactions were predominant in the coating layer formation.

\subsubsection{Magneto-sensitive CS -based Systems}

Magneto-sensitive systems are typically composed of magnetic cores such as $\mathrm{Fe}_{2} \mathrm{O}_{3}$ or $\mathrm{Fe}_{3} \mathrm{O}_{4}$, and polymeric shells, thus presenting a strong magnetic response in the presence of an external magnetic field [70]. As drug delivery carriers, these systems could be transferred to the therapeutic site by applying a magnetic field where they can also exhibit significant heating effects. On the other hand, drug-loaded magnetic NPs could be used to selectively accumulate the drug at the target site by an external magnetic field and, therefore, reduce the doses necessary to achieve the therapeutic concentration. In this context, Natesan et al. [76] prepared magnetic NPs of artemisinin using CS by the ionicgelation method for the treatment of breast cancer. The NPs were smooth and spherical in nature and their size was in the range of $349-445 \mathrm{~nm}$. They showed $55 \%$ to $62.5 \%$ of drug encapsulation efficiency and $20 \%$ to $25 \%$ drug loading capacity. Around $62 \%$ to $78 \%$ of artemisinin was released from the artemisinin magnetic NPs over the period of $48 \mathrm{~h}$. Finally, on the application of physiologically acceptable external magnetic field, FITC conjugated artemisinin magnetic NPs showed an enhanced accumulation of NPs in the 4T1 breast tumour tissues of BALB/c mice model.

\subsection{CS-based Nanodelivery Systems}

The trend of micronizing carrier polymer particles began before the introduction of nanotechnology into drug delivery systems. NPs have a size of $100 \mathrm{~nm}$ or less and are being used intensively in innovative commercial products, including the development of nanotechnological platforms for medicinal applications. The applicability of NPs for use in drug delivery depends on many parameters, such as size and porosity. The size of the NPs determines the specific surface area of the drug, improving the rate of dissolution when the area increases. On the other hand, porosity is an important property because it determines, for example, the ability to trap gases in the NPs. This property can be used to control the release rate of the drug and to direct it to specific regions of the body. NPs can be used to deliver drugs through several administration routes and, for example, have been successfully proved to deliver intravenous drugs, ensuring safe passage through the smallest blood vessels in the body.

Undoubtedly, one of the greatest challenges in medicine is to combine emerging nanotechnology with cellular and molecular techniques in order to develop better diagnostics and drug delivery systems to treat diseases such as cancer.

CS was introduced as a potential gene carrier in 1995 and various nanosystems based on CS and derivatives have been investigated, including unmodified CS with different MW and DD, quaternized CS, bile acid-modified CS, PEGylated CS, and CS bearing specific ligands [77]. CS-based nanodelivery systems have been extensively studied for organspecific applications (e.g., for colon, kidney, lung, and liver) [16].

An interesting approach was carried out by Li et al. [78], who designed CS-based multifunctional nanocarriers modified by L-valine, used as a target ligand to facilitate the absorption of the small intestine, and phenylboronic acid, used as a glucose-responsive unit, to overcome multiple barriers associated to the oral delivery of insulin. The results showed that the nanocarriers exhibited low toxicity and excellent stability in the protein solution. Moreover, the nanocarriers presented protective properties for insulin against enzymatic degradation. After oral administration to diabetic rats, the insulin-loaded nanocarriers exhibited an obvious hypoglycemic effect and, in addition, higher serum insulin level could be obtained after post-administration and maintained a higher level for a longer time. All these results suggested that insulin-loaded CS-based nanocarriers have a potential application in diabetes treatment via oral ingestion.

\subsection{CS-based Systems for Gene Delivery}

In recent years, the appearance of gene therapy as an emerging trend in medicine has been evident due to its great therapeutic potential to develop curative treatments for numerous diseases of genetic origin. Gene therapy is based on the transfer of genetic material to specific cells of the body to regulate the expression of certain proteins. However, the systemic delivery of naked therapeutic genes is unrealistic because of their non-specificity toward target cells, rapid clearance by the mononuclear phagocyte system, and vulnerability to nuclease degradation [79]. Additionally, the large size, hydrophilic nature, and high anionic charge density can also hinder intracellular delivery of the naked gene [80]. Thus, the main challenge of gene therapy in human medicine is the development of gene delivery vectors, in order to help the process of transfer of the therapeutic gene to the target cells to be carried out safely and efficiently. This has motivated researchers to look for potential alternatives for gene transfer vectors. In general, vectors are classified into two groups: viral and non-viral. According to this classification, the polymeric systems of genes delivery are included in the category of non-viral vectors. A non-viral vector must not only allow reaching the target action site in order to achieve a local or systemic effect, but must also facilitate intracellular uptake and improve the intracellular traffic of the nucleic acid payload. CS is one of the most promising among natural polymer-based gene delivery vectors, due to its biodegradability, biocompatibility, and degradation potential [81]. Its role in gene delivery is supported by its ability to protonate in acidic solution and to form a complex with DNA through electrostatic interactions [82]. The stability and transfection efficiency of CS/DNA polyplexes are strongly influenced by various formulation parameters, 
which include MW and DD of CS, stoichiometry or N/P ratio of the CS/DNA polyplex (charge ratio of amines of CS to phosphates of DNA), $\mathrm{pH}$ of the transfection medium, serum concentration, and cell types [83, 84]. The modification of these parameters can significantly influence particle size, surface charge, stability, cellular uptake, and dissociation of CS/DNA polyplex, and hence the overall gene transfection efficiency. Therefore, these parameters must be considered carefully while designing CS-based gene delivery vectors.

In 1995, CS was first described as a delivery system for plasmids by Mumper et al. [77]. One of the major challenges in gene delivery is to protect DNA from nuclease action. The cationic charge of CS is found to be very useful to interact ionically with negatively charged DNA, thereby protecting the DNA against nuclease activity [85]. CS/DNA NPs may be readily formed by coacervation between the positively charged amine groups on CS and negatively charged phosphate groups on DNA.

In an interesting study performed by Amaduzzi et al. [86], the observed aggregation behavior of CS/DNA complexes was compared with the predictions of existing models for the complexation of oppositely charged polyelectrolytes. CS/DNA complexes were prepared at different charge ratios and CS MW and their structure was characterized using different and complementary microscopy approaches, light scattering and electrophoretic mobility techniques. Distinctive "tadpole-like" aggregates were observed when the polycation was in excess, whereas only globular aggregates were found in excess of DNA. Close to the isoelectric point, elongated fiber-like structures appeared. With regard to the models discussed, different apparently uncorrelated observations reported in the literature found a systematic interpretation, concluding that these models are useful tools to guide the design of new and more efficient polycation-based vectors for more effective gene delivery.

Boonthum et al. [87] synthesized a gonadotropinreleasing hormone-conjugated $\mathrm{CS}(\mathrm{GnRH}-\mathrm{CS})$ as a potential vector for gene delivery. They found that the GnRH-CS specifically delivered plasmid DNA to targeted cells and exhibited higher transfection activity compared to unmodified CS. They concluded that GnRH-CS could be a promising carrier for targeted DNA delivery to GnRHR-expressing cells.

Other study performed by Lee et al. [88] describes the synthesis of glycol CS-methyl acrylate-polyethylenimine (GMP) for use as a non-viral vector system. The obtained materials were in vitro evaluated for application as gene delivery vehicles, showing low cytotoxicity and high transfection efficiency. The results allowed the authors concluding that GMP polymer could be appropriate for non-viral gene delivery vector.

\subsection{CS-based Systems in Cancer Therapy}

Therapeutics against cancer is an area that requires continuous improvement of the drugs to achieve greater therapeutic efficacy and reduce side-effects to minimum thresholds. Chemotherapy is based mainly on the use of alkylating agents, antimetabolites, anthracyclines, alkanoids and terpenoids, and is generally administered by intravenous injection. There are two groups of substances which are used for anticancer pharmacotherapy: small cytostatic drugs like carboplatin, doxorubicin, and 5-fluorouracil (5-FU), and nucleic acid- or peptide-based actives. Although all of these substances are highly effective in vitro when applied directly to cell cultures, their clinical use and their therapeutic efficacy are limited by either severe side effects or insufficient bioavailability. Nevertheless, the main challenge is that most of the chemotherapeutic agents lack an intrinsic specificity for the malignant cells, affecting other normal cells. In addition, many of these drugs have poor aqueous solubility or cannot reach tumor tissue due to cellular mechanisms causing drugs resistance. For this reason, effective polymeric drug carriers are needed to overcome these drawbacks.

In this context, CS has been frequently used as an excipient in such systems and proved to be an excellent alternative. In addition, CS is recognized as a substance that helps improve the cellular uptake and tissue penetration of different active agents. Furthermore, this biopolymer has various biological effects by itself, such as antimicrobial activity, stimulation of the immune system and even some activity against cancer, since it can modify tumor growth, what gives an additional value to its use as a promising excipient for specific therapeutic strategies. Various researches demonstrate the beneficial effect of CS in the transport of chemotherapeutic drugs, such as doxorubicin and cisplatin $[89,90]$. CS also stands out for its versatility, since in a drug delivery system it can be used as an oligo or polymer, as a polymer-drug complex, and as a material for the formation of hydrogel or for the production of micro or nanoparticles. CS properties can be easily modulated by chemical modifications through the amino groups, using, for example, side carbon chains or functional groups. CS-based carrier systems can accumulate inside the malignant tissue, due to the so-called enhanced permeability and retention effect, caused by leaky blood vessel endothelia in the tumor. Thus, the side effects of the active agents in healthy tissues are reduced while the drug concentration on the site of action is increased. CS formulations enable anticancer drugs to overcome their biopharmaceutical inadequateness and enhance their biological activity.

Nanotechnology has allowed great advances in the detection and treatment of cancer. As current technologies are refined and improved, the side effects inherent in cancer treatment will diminish, allowing patients to have a better life quality and longer survival. Similarly, it can be predicted that there will be notable advances in the development of CS-based NP delivery systems for the treatment of different human diseases, with special emphasis on the treatment of cancer. Therefore, this biopolymer can play a key role in the investigation of new therapeutic strategies and offers valuable advantages in the development of carrier systems for application in local and systemic therapy against cancer. Tan et al. [91] published a review article about CS-based delivery systems of doxorubicin for cancer therapy. They summarized different methods for the drug encapsulation to deliver it in the specific lesion site, which allowed reducing side-effects.

Recently, Poloxamer surface modified trimethyl CS NPs were prepared by $\mathrm{Li}$ et al. [92] for the delivery of methotrexate (MTX) in osteosarcoma. The results indicated 
that the NPs presented higher cytotoxicity in MG63 cells than the free MTX, explained by its enhanced cellular uptake through energy-dependent endocytosis process. Furthermore, the apoptosis effect was also increased in comparison to that of free drug, with bright chromatin condensation and nuclear fragmentation.

On the other hand, Rudzinski et al. [93] encapsulated anti- $\beta$-catenin small interfering RNA in CS and PEG-grafted CS NPs, for transfection into colon cancer cells. They found a decrease in $\beta$-catenin protein levels in cultured colon cancer cells for both CS and PEG-grafted CS NPs.

\section{FUTURE TRENDS}

Over the last few years, scientific databases reveal that there are thousands of articles and patents related to chitin and CS-based materials for biomedical applications. As CS biodegradable properties, low immunogenicity, bioactivity and enhanced biocompatibility, it can be considered the firstchoice polymer to be employed in the biomedical area.

Probably, the most promising medical application that should be emphasized is its use in drug delivery systems. Since CS and derivatives systems allow higher drug loads, they are able to modulate the drug release, and matrices with different degradation kinetics can be designed. In addition, CS-based formulations could be designed to respond to external stimuli, which make it possible to produce intelligent and effective drug delivery systems.

Other applications that remain to be explored in depth are the possibility of linking CS, through covalent or ionic bonds, to proteins and peptides in scaffolds for tissue regeneration, and as carriers in the administration of genes, due to the cationic nature of $\mathrm{CS}$ and its ability to form chelates with DNA.

In most of the biomedical applications mentioned, a preclinical and clinical test of the designed systems were not carried out, which is fundamental for their application as new devices in clinical therapy.

Future efforts should focus on other important aspects. On one hand, the research and development of CS and derivatives with improved solubility must continue. Precisely, its low solubility is what restricts many applications of this biomaterial. On the other hand, since the MW, DD, polymerization degree and obtaining source govern CS behavior, it is necessary to standardize the production methods to guarantee reproducibility of CS physicochemical characteristics among batches. For this reason, optimizing the processes of isolation and purification of CS to improve purity and reproducibility according to international Pharmacopoeias will be critical for the commercial success of CSbased formulations. Biotechnological processes or genetic engineering methods, focused on producing a material with previously designed characteristics, are some alternatives to those currently obtained from natural marine sources.

\section{CONCLUSION}

Numerous developments have been reported on CS derivatives with promising properties, such as better solubility, stronger mucoadhesive capacities, and inhibitory enzymatic properties. The qualities of CS related to the improvement of the permeation, its mucoadhesive properties, as well as the controlled release of drugs and the ability to open tight junctions, allow to conclude that this biopolymer will be an excellent tool for the development of systems for peptide delivery. Certainly, these properties can be further improved by simple chemical modifications in their primary amino group. These unique qualities make CS and, in particular, its derivatives, valuable excipients for the development of drug delivery systems for the treatment of various human pathologies and other applications. Despite all the qualities of this polymer, there are only few CS-based products in the market, so it is a priority to enhance the research to develop new technologies and CS-based systems to enforce this biopolymer in the industry.

\section{LIST OF ABBREVIATIONS}

5-FU $=$-fluorouracil
CS $=$ Chitosan
DD $=$ Deacetylation Degree
DNA $=$ Deoxyribonucleic Acid
FDA $=$ Food and Drug Administration
GI $=$ Gastrointestinal
GMP $=$ CS-methyl Acrylate-polyethylenimine
GnRH $=$ Gonadotropin-releasing Hormone
KCSS $=$ Kollicoal
LT $=$ Ludiflash
MT
MTX Microcrystalline Cellulose
MW $=$ Methotrexate
NP $=$ Nolecular Weight
PEG $=$ Polyethylene Glycol
PVA $=$ Polyvinyl Alcohol
QUR $=$ Quercetin
RES $=$ Resveratrol
RF $=$ Radio Frequency
RNA $=$ Ribonucleic Acid
TMC $=$-trimethyl Chitosan

\section{CONSENT FOR PUBLICATION}

Not applicable.

\section{FUNDING}

None.

\section{CONFLICT OF INTEREST}

The authors declare no conflict of interest, financial or otherwise. 


\section{ACKNOWLEDGEMENTS}

The authors would like to thank: CIUNSa (Argentine, Grant 2277, 2457 and 2146/0) for financial support, and CONICET (Argentine) and CIN (Argentine) for the research fellowships.

\section{REFERENCES}

[1] Fiamingo, A.; Delezuk, J.A.d.M.; Trombotto, S.; David, L.; Campana-Filho, S.P. Extensively deacetylated high molecular weight chitosan from the multistep ultrasound-assisted deacetylation of beta-chitin. Ultrasonics Sonochem., 2016, 32, 79-85.

[2] Elizabeth, B. Alogenosis iatrogénica. PhD Thesis, Universidad Católica de Cuenca: Cuenca, Ecuador, 2012.

[3] Kadajji, V.G.; Betageri, G.V. Water soluble polymers for pharmaceutical applications. Polymers, 2011, 3, 1972-2009.

[4] Dutta, P.K.; Dutta, J.; Tripathi, V.S. Chitin and chitosan: chemistry, properties and application. J. Sci. Ind. Res., 2004, 63, 20-31.

[5] Macleod, G.; T Fell, J.; Collett, J. An in vitro investigation into the potential for bimodal drug release from pectin/chitosan/HPMCcoated tablets. Int. J. Pharm., 1999, 188(1), 11-18.

[6] Kokil, S.; Patil, P.; Mahadik, K.; Paradkar, A. Studies on spraydried mixtures of chitosan and hydrolyzed gelatin as tablet binder: A technical note. AAPS PharmSciTech., 2005, 6(3), 437-443.

[7] Chuang, C.Y.; Don, T.M.; Chiu, W.Y. Synthesis and characterization of stimuli-responsive porous/hollow nanoparticles by self-assembly of chitosan-based graft copolymers and application in drug release. J. Polym. Sci. A1, 2010, 48(11), 2377-2387.

[8] Ribeiro, P.A.F.; Dias, D.S.; Lage, D.P.; Costa, L.E.; Martins, V.T.; Tavares, G.S.V.; Mendonça, D.V.C.; Lima, M.P.; Oliveira, J.S.; Steiner, B.T.; Machado-de-Ávila, R.A.; Roatt, B.M.; ChávezFumagalli, M.A.; Menezes-Souza, D.; Duarte, M.C.; Teixeira, A.L.; Coelho, E.A.F. Evaluation of a Leishmania hypothetical protein administered as DNA vaccine or recombinant protein against Leishmania infantum infection and its immunogenicity in humans. Cell. Immunol., 2018, 331, 67-77.

[9] Boateng, J.S.; Matthews, K.H.; Stevens, H.N.E.; Eccleston, G.M. Wound Healing Dressings and Drug Delivery Systems: A Review. J. Pharm. Sci., 2008, 97(8), 2892-2923.

[10] Singla, A.K.; Chawla, M. Chitosan: some pharmaceutical and biological aspects-an update. J. Pharm. Pharmacol., 2001, 53(8), 1047-67.

[11] Muzzarelli, R.A.A.; Muzzarelli, C. Chitosan chemistry: Relevance to the biomedical sciences. In: Polysaccharides I: Structure, Characterization and Use; Heinze, T., Ed.; Springer Berlin, Heidelberg, 2005; Vol. 186, pp 151-209.

[12] Englehart, M.S.; Cho, S.D.; Tieu, B.H.; Morris, M.S.; Underwood, S.J.; Karahan, A.; Muller, P.J.; Differding, J.A.; Farrell, D.H.; Schreiber, M.A. A novel highly porous silica and chitosan-based hemostatic dressing is superior to HemCon and gauze sponges. $J$. Trauma, 2008, 65(4), 884-90; discussion 890-2.

[13] Malmquist, J.P.; Clemens, S.C.; Oien, H.J.; Wilson, S.L. Hemostasis of oral surgery wounds with the HemCon Dental Dressing. $J$. Oral Maxill. Surg., 2008, 66(6), 1177-83.

[14] Brown, M.A.; Daya, M.R.; Worley, J.A. Experience with chitosan dressings in a civilian EMS system. J. Emerg. Med., 2009, 37(1), 17.

[15] Landriscina, A.; Rosen, J.; Friedman, A.J. Biodegradable chitosan nanoparticles in drug delivery for infectious disease. Nanomedicine (Lond), 2015, 10(10), 1609-19.

[16] Park, J.H.; Saravanakumar, G.; Kim, K.; Kwon, I.C. Targeted delivery of low molecular drugs using chitosan and its derivatives. Adv. Drug Deliv. Rev., 2010, 62(1), 28-41.

[17] Gan, Q.; Wang, T. Chitosan nanoparticle as protein delivery carrier-systematic examination of fabrication conditions for efficient loading and release. Colloids Surf. B Biointerfaces, 2007, 59(1), 24-34.

[18] Illum, L.; Jabbal-Gill, I.; Hinchcliffe, M.; Fisher, A.N.; Davis, S.S. Chitosan as a novel nasal delivery system for vaccines. Adv. Drug Deliv. Rev., 2001, 51(1-3), 81-96.

[19] Mao, S.; Sun, W.; Kissel, T. Chitosan-based formulations for delivery of DNA and siRNA. Adv. Drug Deliv. Rev., 2010, 62(1), 1227.
[20] Galván Márquez, I.; Akuaku, J.; Cruz, I.; Cheetham, J.; Golshani, A.; Smith, M.L. Disruption of protein synthesis as antifungal mode of action by chitosan. Int. J. Food Microbiol., 2013, 164(1), 108112.

[21] Kong, M.; Chen, X.G.; Xing, K.; Park, H.J. Antimicrobial properties of chitosan and mode of action: A state of the art review. Int. $J$ Food Microbiol., 2010, 144(1), 51-63.

[22] Badawy, M.E.I.; Rabea, E.I. A biopolymer chitosan and its derivatives as promising antimicrobial agents against plant pathogens and their applications in crop protection. Int. J. Carbohydr. Chem., 2011, 1, 1-29.

[23] Gamal, R.F.; El-Tayeb, T.S.; Raffat, E.I.; Ibrahim, H.M.M.; Bashandy, A.S. Optimization of chitin yield from shrimp shell waste by Bacillus subtilis and impact of gamma irradiation on production of low molecular weight chitosan. Int. J. Biol. Macromol., 2016, 91, 598-608.

[24] Percot, A.; Viton, C.; Domard, A. Optimization of chitin extraction from shrimp shells. Biomacromolecules, 2003, 4(1), 12-8.

[25] Crofton, A.R.; Hudson, S.M.; Howard, K.; Pender, T.; Abdelgawad, A.; Wolski, D.; Kirsch, W.M. Formulation and characterization of a plasma sterilized, pharmaceutical grade chitosan powder. Carbohydr. Polym., 2016, 146, 420-426.

[26] Jayakumar, R.; Prabaharan, M.; Reis, R.L.; Mano, J.F. Graft copolymerized chitosan-present status and applications. Carbohydr. Polym., 2005, 62(2), 142-158.

[27] Harris, M.; Alexander, C.; Wells, C.M.; Bumgardner, J.D.; Carpenter, D.P.; Jennings, J.A. Chitosan for the delivery of antibiotics. In: Chitosan Based Biomaterials Jennings, J. A.; Bumgardner, J. D., Eds.; Woodhead Publishing: 2017; Vol. 2, pp 147-173.

[28] Ramasamy, P.; Subhapradha, N.; Thinesh, T.; Selvin, J.; Selvan, K.M.; Shanmugam, V.; Shanmugam, A. Characterization of bioactive chitosan and sulfated chitosan from Doryteuthis singhalensis (Ortmann, 1891). Int. J. Biol. Macromol., 2017, 99, 682-691.

[29] Gierszewska, M.; Ostrowska-Czubenko, J. Chitosan-based membranes with different ionic crosslinking density for pharmaceutical and industrial applications. Carbohydr. Polym., 2016, 153, 501511.

[30] Jóźwiak, T.; Filipkowska, U.; Szymczyk, P.; Rodziewicz, J.; Mielcarek, A. Effect of ionic and covalent crosslinking agents on properties of chitosan beads and sorption effectiveness of Reactive Black 5 dye. React. Funct. Polym., 2017, 114, 58-74.

[31] Maslakci, N.N.; Ulusoy, S.; Oksuz, A.U. Investigation of the effects of plasma-treated chitosan electrospun fibers onto biofilm formation. Sensor. Actuator., 2017, 246, 887-895.

[32] Illum, L. Chitosan and its use as a pharmaceutical excipient. Pharm. Res., 1998, 15(9), 1326-31.

[33] Drechsler, M.; Garbacz, G.; Thomann, R.; Schubert, R. Development and evaluation of chitosan and chitosan/Kollicoat ${ }^{\circledR}$ Smartseal $30 \mathrm{D}$ film-coated tablets for colon targeting. Eur. J. Pharm. Biopharm., 2014, 88(3), 807-815.

[34] Upadrashta, S.M.; Katikaneni, P.R.; Nuessle, N.O. Chitosan as a tablet binder. Drug Dev. Ind. Pharm., 1992, 18(15), 1701-1708.

[35] Movaffagh, J.; Ghodsi, A.; Fazly Bazzaz, B.S.; Sajadi Tabassi, S.A.; Ghodrati Azadi, H. The use of natural biopolymer of chitosan as biodegradable beads for local antibiotic delivery: release studies. Jundishapur J. Nat. Pharm. Prod., 2013, 8(1), 27-33.

[36] Sogias, I.A.; Williams, A.C.; Khutoryanskiy, V.V. Why is chitosan mucoadhesive? Biomacromolecules, 2008, 9(7), 1837-1842.

[37] Gonçalves, I.; Henriques, P.; Seabra, C.; Martins, M.C. The potential utility of chitosan micro/nanoparticles in the treatment of gastric infection. Expert Rev. Anti Infect. Ther., 2014, 12(8), 981-992.

[38] Wang, M.; Liu, M.; Xie, T.; Zhang, B.-F.; Gao, X.-L. Chitosanmodified cholesterol-free liposomes for improving the oral bioavailability of progesterone. Colloids Surf. B Biointerfaces, 2017, 159, 580-585.

[39] Ubaid, M.; Murtaza, G. Fabrication and characterization of genipin cross-linked chitosan/gelatin hydrogel for $\mathrm{pH}$-sensitive, oral delivery of metformin with an application of response surface methodology. Int. J. Biol. Macromol., 2018, 114, 1174-1185.

[40] S., P.; Y., G. Polymers in mucoadhesive buccal drug delivery system - A review. Int. J. Res. Pharm. Sci., 2010.

[41] Freag, M.S.; Saleh, W.M.; Abdallah, O.Y. Exploiting polymer blending approach for fabrication of buccal chitosan-based composite sponges with augmented mucoadhesive characteristics. Eur. J. Pharm. Sci., 2018, 120, 10-19. 
[42] Portero, A.; Teijeiro-Osorio, D.; Alonso, M.J.; Remuñán-López, C. Development of chitosan sponges for buccal administration of insulin. Carbohydr. Polym., 2007, 68(4), 617-625.

[43] Tejada, G.; Barrera, M.G.; Piccirilli, G.N.; Sortino, M.; Frattini, A.; Salomon, C.J.; Lamas, M.C.; Leonardi, D. Development and evaluation of buccal films based on chitosan for the potential treatment of oral candidiasis. AAPS PharmSciTech., 2017, 18(4), 936-946.

[44] Moes, A.J. Gastroretentive dosage forms. Crit. Rev. Ther. Drug Carrier Syst., 1993, 10(2), 143-95.

[45] Yang, L.; Eshraghi, J.; Fassihi, R. A new intragastric delivery system for the treatment of Helicobacter pylori associated gastric ulcer: in vitro evaluation. J. Control. Release, 1999, 57(3), 215-22.

[46] Praveen, R.; Prasad Verma, P.R.; Venkatesan, J.; Yoon, D.-H.; Kim, S.-K.; Singh, S.K. In vitro and in vivo evaluation of gastroretentive carvedilol loaded chitosan beads using Gastroplus ${ }^{\mathrm{TM}}$. Int. J. Biol. Macromol., 2017, 102, 642-650.

[47] Kim, S.; Jo, A.; Ahn, J. Application of chitosan-alginate microspheres for the sustained release of bacteriophage in simulated gastrointestinal conditions. Int. J. Food Sci. Tech., 2015, 50(4), 913918

[48] Abruzzo, A.; Bigucci, F.; Cerchiara, T.; Saladini, B.; Gallucci, M.C.; Cruciani, F.; Vitali, B.; Luppi, B. Chitosan/alginate complexes for vaginal delivery of chlorhexidine digluconate. Carbohydr. Polym., 2013, 91(2), 651-658.

[49] Marciello, M.; Rossi, S.; Caramella, C.; Remuñán-López, C. Freeze-dried cylinders carrying chitosan nanoparticles for vaginal peptide delivery. Carbohydr. Polym., 2017, 170, 43-51.

[50] Dabaghian, M.; Latifi, A.M.; Tebianian, M.; NajmiNejad, H.; Ebrahimi, S.M. Nasal vaccination with r4M2e.HSP70c antigen encapsulated into N-trimethyl chitosan (TMC) nanoparticulate systems: Preparation and immunogenicity in a mouse model. Vaccine, 2018, 36(20), 2886-2895.

[51] Agnihotri, S.A.; Mallikarjuna, N.N.; Aminabhavi, T.M. Recent advances on chitosan-based micro- and nanoparticles in drug delivery. J. Control. Release, 2004, 100(1), 5-28.

[52] Luppi, B.; Bigucci, F.; Abruzzo, A.; Corace, G.; Cerchiara, T.; Zecchi, V. Freeze-dried chitosan/pectin nasal inserts for antipsychotic drug delivery. Eur. J. Pharm. Biopharm., 2010, 75(3), 381387.

[53] Huang, Y.C.; Vieira, A.; Huang, K.L.; Yeh, M.K.; Chiang, C.H. Pulmonary inflammation caused by chitosan microparticles. $J$. Biomed. Mater. Res. A, 2005, 75A(2), 283-287.

[54] Zhang, W.F.; Zhao, X.T.; Zhao, Q.S.; Zha, S.H.; Liu, D.M.; Zheng, Z.J.; Li, W.T.; Zhou, H.Y.; Yan, F. Biocompatibility and characteristics of theophylline/carboxymethyl chitosan microspheres for pulmonary drug delivery. Polym. Int., 2014, 63(6), 1035-1040.

[55] Manca, M.L.; Manconi, M.; Valenti, D.; Lai, F.; Loy, G.; Matricardi, P.; Fadda, A.M. Liposomes coated with chitosan-xanthan gum (chitosomes) as potential carriers for pulmonary delivery of rifampicin. J. Pharm. Sci., 2012, 101(2), 566-575.

[56] Oyarzun-Ampuero, F.A.; Brea, J.; Loza, M.I.; Torres, D.; Alonso, M.J. Chitosan-hyaluronic acid nanoparticles loaded with heparin for the treatment of asthma. Int. J. Pharm., 2009, 381(2), 122-9.

[57] Anirudhan, T.S.; Nair, S.S.; Nair, A.S. Fabrication of a bioadhesive transdermal device from chitosan and hyaluronic acid for the controlled release of lidocaine. Carbohydr. Polym., 2016, 152, 687698.

[58] Cui, Z.; Zheng, Z.; Lin, L.; Si, J.; Wang, Q.; Peng, X.; Chen, W. Electrospinning and crosslinking of polyvinyl alcohol/chitosan composite nanofiber for transdermal drug delivery. Adv. Polym. Tech., 2018, 37(6), 1917-1928.

[59] De Campos, A.M.; Sanchez, A.; Alonso, M.J. Chitosan nanoparticles: a new vehicle for the improvement of the delivery of drugs to the ocular surface. Application to cyclosporin A. Int. J. Pharm., 2001, 224(1-2), 159-68

[60] Schipper, N.G.; Olsson, S.; Hoogstraate, J.A.; deBoer, A.G.; Varum, K.M.; Artursson, P. Chitosans as absorption enhancers for poorly absorbable drugs 2 : mechanism of absorption enhancement. Pharm. Res., 1997, 14(7), 923-9.

[61] Felt, O.; Furrer, P.; Mayer, J.M.; Plazonnet, B.; Buri, P.; Gurny, R. Topical use of chitosan in ophthalmology: tolerance assessment and evaluation of precorneal retention. Int. J. Pharm., 1999, 180(2), 185-93.
[62] Wadhwa, S.; Paliwal, R.; Paliwal, S.R.; Vyas, S.P. Nanocarriers in ocular drug delivery: an update review. Curr. Pharm. Des., 2009, 15(23), 2724-50

[63] Natesan, S.; Pandian, S.; Ponnusamy, C.; Palanichamy, R.; Muthusamy, S.; Kandasamy, R. Co-encapsulated resveratrol and quercetin in chitosan and peg modified chitosan nanoparticles: For efficient intra ocular pressure reduction. Int. J. Biol. Macromol., 2017.

[64] Rodrigues, L.B.; Leite, H.F.; Yoshida, M.I.; Saliba, J.B.; Cunha, A.S., Jr.; Faraco, A.A. In vitro release and characterization of chitosan films as dexamethasone carrier. Int. J. Pharm., 2009, 368(12), 1-6.

[65] Bhattarai, N.; Gunn, J.; Zhang, M. Chitosan-based hydrogels for controlled, localized drug delivery. Adv. Drug Deliv. Rev., 2010, 62(1), 83-99

[66] Jayakumar, R.; Menon, D.; Manzoor, K.; Nair, S.V.; Tamura, H. Biomedical applications of chitin and chitosan based nanomaterials-A short review. Carbohydr. Polym., 2010, 82(2), 227-232.

[67] Lai, W.F.; Lin, M.C. Nucleic acid delivery with chitosan and its derivatives. J. Control. Release, 2009, 134(3), 158-68.

[68] Buschmann, M.D.; Merzouki, A.; Lavertu, M.; Thibault, M.; Jean, M.; Darras, V. Chitosans for delivery of nucleic acids. Adv. Drug Deliv. Rev., 2013, 65(9), 1234-70.

[69] Amidi, M.; Romeijn, S.G.; Verhoef, J.C.; Junginger, H.E.; Bungener, L.; Huckriede, A.; Crommelin, D.J.; Jiskoot, W. N-trimethyl chitosan (TMC) nanoparticles loaded with influenza subunit antigen for intranasal vaccination: biological properties and immunogenicity in a mouse model. Vaccine, 2007, 25(1), 144-53.

[70] Li, Y.; Dong, H.; Wang, K.; Shi, D.; Zhang, X.; Zhuo, R. Stimulusresponsive polymeric nanoparticles for biomedical applications. Sci. China Chem., 2010, 53(3), 447-457.

[71] Du, H.; Liu, M.; Yang, X.; Zhai, G. The design of pH-sensitive chitosan-based formulations for gastrointestinal delivery. Drug Discov. Today, 2015, 20(8), 1004-1011.

[72] Yilmaz, E.; Yalinca, Z.; Yahya, K.; Sirotina, U. pH responsive graft copolymers of chitosan. Int. J. Biol. Macromol., 2016, 90, 6874

[73] Li, Z.; Shim, H.; Cho, M.O.; Cho, I.S.; Lee, J.H.; Kang, S.-W.; Kwon, B.; Huh, K.M. Thermo-sensitive injectable glycol chitosanbased hydrogel for treatment of degenerative disc disease. Carbohydr. Polym., 2018, 184, 342-353.

[74] Jeong, Y.-I.L.; Cha, B.; Lee, H.L.; Song, Y.H.; Jung, Y.H.; Kwak, T.W.; Choi, C.; Jeong, G.-W.; Nah, J.W.; Kang, D.H. Simple nanophotosensitizer fabrication using water-soluble chitosan for photodynamic therapy in gastrointestinal cancer cells. Int. J. Pharmaceut., 2017, 532(1), 194-203.

[75] Goycoolea, F.M.; Milkova, V. Electrokinetic behavior of chitosan adsorbed on o/w nanoemulsion droplets. Colloids Surf. A, 2017, 519, 205-211.

[76] Natesan, S.; Ponnusamy, C.; Sugumaran, A.; Chelladurai, S Shanmugam Palaniappan, S.; Palanichamy, R. Artemisinin loaded chitosan magnetic nanoparticles for the efficient targeting to the breast cancer. Int. J. Biol. Macromol., 2017, 104, 1853-1859.

[77] Mumper, R.J.; Wang, J.; Claspell, J.M.; Rolland, A.P. Novel polymeric condensing carriers for gene delivery. P. Controll. Release Soc., 1995, (22)

[78] Li, L.; Jiang, G.; Yu, W.; Liu, D.; Chen, H.; Liu, Y.; Tong, Z. Kong, X.; Yao, J. Preparation of chitosan-based multifunctional nanocarriers overcoming multiple barriers for oral delivery of insulin. Mater. Sci. Eng. C Mater. Biol. Appl. , 2017, 70, 278-286.

[79] Kim, T.-H.; Jiang, H.-L.; Jere, D.; Park, I.-K.; Cho, M.-H.; Nah, J.W.; Choi, Y.-J.; Akaike, T.; Cho, C.-S. Chemical modification of chitosan as a gene carrier in vitro and in vivo. Prog. Polvm. Sci., 2007, 32(7), 726-753.

[80] Tamboli, V.; Mishra, G.P.; Mitra, A.K. Polymeric vectors for ocular gene delivery. Ther. deliv., 2011, 2(4), 523-536.

[81] Prabaharan, M.; Mano, J.F. Chitosan-based particles as controlled drug delivery systems. Drug Deliv., 2005, 12(1), 41-57.

[82] Duceppe, N.; Tabrizian, M. Advances in using chitosan-based nanoparticles for in vitro and in vivo drug and gene delivery. Expert Opin. Drug Deliv., 2010, 7(10), 1191-207.

[83] Ishii, T.; Okahata, Y.; Sato, T. Mechanism of cell transfection with plasmid/chitosan complexes. BBA-Biomembranes, 2001, 1514(1), 51-64. 
[84] Kiang, T.; Wen, J.; Lim, H.W.; Leong, K.W. The effect of the degree of chitosan deacetylation on the efficiency of gene transfection. Biomaterials, 2004, 25(22), 5293-301.

[85] Senel, S.; J McClure, S. Potential applications of chitosan in veterinary medicine. Adv. Drug Deliv. Rev., 2004, 56, 1467-1480.

[86] Amaduzzi, F.; Bomboi, F.; Bonincontro, A.; Bordi, F.; Casciardi, S.; Chronopoulou, L.; Diociaiuti, M.; Mura, F.; Palocci, C.; Sennato, S. Chitosan-DNA complexes: Charge inversion and DNA condensation. Colloids Surf. B Biointerfaces, 2014, 114, 1-10.

[87] Boonthum, C.; Namdee, K.; Boonrungsiman, S.; Chatdarong, K.; Saengkrit, N.; Sajomsang, W.; Ponglowhapan, S.; Yata, T. Chitosan-based DNA delivery vector targeted to gonadotropin-releasing hormone (GnRH) receptor. Carbohydr. Polym., 2017, 157, 311320.

[88] Lee, Y.H.; Park, H.I.; Choi, J.S. Novel glycol chitosan-based polymeric gene carrier synthesized by a Michael addition reaction with low molecular weight polyethylenimine. Carbohydr. Polym., 2016, 137, 669-677.
[89] Feng, C.; Sun, G.; Wang, Z.; Cheng, X.; Park, H.; Cha, D.; Kong, M.; Chen, X. Transport mechanism of doxorubicin loaded chitosan based nanogels across intestinal epithelium. Eur. J. Pharm. Biopharm., 2014, 87(1), 197-207.

[90] Moura, M.; Gil, M.; Figueiredo, M. Delivery of cisplatin from thermosensitive co-cross-linked chitosan hydrogels. Eur. Polym. J. 2013, 49(9), 2504-2510

[91] Tan, M.L.; Choong, P.F.; Dass, C.R. Review: doxorubicin delivery systems based on chitosan for cancer therapy. J. Pharm. Pharmacol., 2009, 61(2), 131-42.

[92] Li, S.; Xiong, Y.; Zhang, X. Poloxamer surface modified trimethyl chitosan nanoparticles for the effective delivery of methotrexate in osteosarcoma. Biomed. Pharmacother., 2017, 90, 872-879.

[93] Rudzinski, W.E.; Palacios, A.; Ahmed, A.; Lane, M.A.; Aminabhavi, T.M. Targeted delivery of small interfering RNA to colon cancer cells using chitosan and PEGylated chitosan nanoparticles. Carbohydr. Polym., 2016, 147, 323-332. 